\title{
NUEVOS DATOS PARA LA DISCUSIÓN ARQUEOLÓGICA DE CORREDORES EN EL BOSQUE DEL SUROESTE DE LA PROVINCIA DE SANTA CRUZ, ARGENTINA: EL CASO DEL ARROYO LOS LOROS
}

\author{
FLAVIA CARBALLO MARINA ${ }^{\mathrm{a}}$, JUAN BAUTISTA BELARDI ${ }^{\mathrm{b}}$ \\ \& LUIS ALBERTO BORREROc
}

\section{RESUMEN}

Los materiales arqueológicos registrados sobre el arroyo Los Loros, Área de La Primavera (La Escondida), en campos de la Ea. Cancha Carrera suman nueva evidencia a la discusión de la existencia de corredores en el bosque del Suroeste de la provincia de Santa Cruz, Argentina. Así, se aporta información sobre el uso por parte de las poblaciones cazadoras recolectoras de un espacio ubicado entre la Cordillera Oriental (por donde discurre el límite con Chile) y la Cordillera Chica y la Meseta Latorre, en el actual ecotono bosque-estepa. Se postula que el valle del arroyo Los Loros habría actuado como un corredor que conecta el valle del río Guillermo con las cabeceras del río Gallegos. La información recuperada concuerda con lo conocido sobre la arqueología de otros sectores de bosque del Sur de Santa Cruz, caracterizados por una baja intensidad de uso estacional y el empleo de rocas disponibles localmente.

PALABRAS CLAVE: corredores, bosque, uso estacional, materias primas líticas.

\section{NEW DATA FOR AN ARCHAEOLOGICAL DISCUSSION OF FOREST CORRIDORS IN THE SOUTHWEST OF SANTA CRUZ PROVINCE, ARGENTINE: THE CASE OF ARROYO LOS LOROS}

\section{ABSTRACT}

A record of archaeological remains at Arroyo Los Loros, La Primavera (La Escondida), Ea. Cancha Carrera is presented. This evidence sustains the existence of forest corridors in Southwest Santa Cruz Province, Argentina. Information on the use of the current forest-steppe ecotone by past hunter-gatherer populations between the Cordillera Oriental (the international border line with Chile) and the Cordillera Chica and Meseta Latorre is provided. It is postulated that the Arroyo

a Universidad Nacional de la Patagonia Austral. ICASUR. Campus Universitário. Piloto "Lero" Rivera s/n. (9400). Rio Gallegos, Santa Cruz, Argentina. flaviacarballomarina@gmail.com

b Universidad Nacional de la Patagonia Austral. ICASUR. CONICET. Campus Universitário. Piloto "Lero" Rivera s/n. (9400) Rio Gallegos, Santa Cruz, Argentina. juanbautistabelardi@gmail.com

c Universidad de Buenos Aires. IMHICIHU-CONICET. Saavedra 15, piso 5 (1083 ACA). Ciudad Autónoma de Buenos Aires, Argentina. laborrero2003@yahoo.com 
Los Loros valley would have been a corridor, connecting the San Guillermo River Valley with the Gallegos River headwaters. This data is in accordance with what is known from the archaeology of other forested sectors in Southern Santa Cruz, all characterized by a low intensity use and the exploitation of locally available lithics.

KEY WORDS: corridors, forest, seasonal use, lithic raw materials.

\section{INTRODUCCIÓN}

De manera reciente Pallo y Borrero (2015) dieron a conocer los resultados de una prospección arqueológica concebida desde una perspectiva distribucional focalizada sobre el valle del río Guillermo, en el Suroeste de la provincia de Santa Cruz (Fig. 1). Se estudiaron las distribuciones artefactuales y se las vinculó con la circulación de las poblaciones cazadoras recolectoras en ambientes de bosque y con un uso estacional. Esto se planteó sobre la base de las mayores densidades artefactuales registradas hacia el occidente y a la manufactura de artefactos empleando bloques de lutita negra de disponibilidad local. A ello se suma, si bien en baja frecuencia, la presencia de módulos laminares. Así, desde el Oeste, el sector del río Guillermo sería integrado en forma logística a los rangos de acción de los grupos humanos. El valle del río habría funcionado como un corredor de bajo costo de circulación que une ambas vertientes de la cordillera. La cronología disponible ubica el uso de este sector hacia el Holoceno tardío, alrededor de 900 años AP (Pallo \& Borrero, 2015), aunque la profundidad temporal hacia el occidente remite al Holoceno medio (Legoupil, 2009).

Aquí se suma nueva evidencia para la discusión de la existencia de corredores a partir del registro de materiales arqueológicos en el valle medio del arroyo Los Loros -también localmente llamado del Oro-, Área de La Primavera (La Escondida), Ea. Cancha Carrera. Este arroyo nace inmediatamente al Sur del curso medio del río Guillermo, corre en dicha dirección entre la Cordillera Oriental (límite internacional) y la Cordillera Chica y la Meseta Latorre, y conecta con las cabeceras de la cuenca del río Gallegos (Fig. 1).

Aunque la evidencia arqueológica se hallan en el ecotono bosque-estepa, en el pasado se habría ubicado en el bosque de ñire (Nothofagus antárctica), que se encuentra actualmente muy cerca (ver Peri et al. 2013; Pallo \& Borrero, 2015). La zona consiste en mosaicos variables de bosque -relictos de una dispersión más amplia- que se emplazan en un paisaje glaciario (García et al. 2014). Las poblaciones locales de cazadores hacían uso del fuego en tiempos históricos tanto para cazar como para abrir espacios de circulación (por ejemplo Ibar Sierra (2002) [1877], p. 141). Además, deben considerarse los cambios antrópicos vinculados con la colonización europea durante los siglos XIX y XX, principalmente la degradación de extensas áreas y actividades de quema. En la zona se registran extensas lentes subsuperficiales de carbones, tocones de árboles -muchas veces quemados- y acumulaciones de madera muerta que atestiguan la reciente presencia de cobertura boscosa. En parte por este motivo, la visibilidad arqueológica es muy buena y el potencial de entierro de los restos culturales es alto.

Los resultados también son relevantes en una escala suprarregional porque amplían la información sobre el uso del bosque en el pasado.

\section{METODOLOGÍA y RESULTADOS}

En una terraza reciente labrada sobre un depósito morénico (352-355 msnm) en la margen izquierda del valle medio del arroyo Los Loros, se identificaron materiales arqueológicos líticos sobre una matriz arenosa y a cielo abierto a lo largo de 400 m (Figs. 2 y 3a). Este espacio fue llamado sitio Arroyo Los Loros y, si bien constituye un palimpsesto, se lo segmentó en cuatro hoyadas de deflación y, cuando se reconocieron concentraciones (basadas sobre la frecuencia artefactual), se decidió recolectar el material de acuerdo con ellas. A la vez, se recuperaron los artefactos que se encontraban 
entre las concentraciones. Las primeras tres hoyadas son contiguas, mientras que la cuarta se encuentra a unos $200 \mathrm{~m}$ hacia el Noroeste, siguiendo un estrecho sector denudado (Fig. 2). Se registraron 63 artefactos líticos.

También en una morena (400 msnm) ubicada inmediatamente hacia el Este se recuperaron cinco artefactos en un pequeño bloque errático (Figs. 2 y 3b) y se realizó un hallazgo aislado sobre una superficie de erosión vinculada con otra morena (365 msnm) (Fig. 2). A la vez, en torno a estos espacios se relevaron $35.500 \mathrm{~m}^{2}$ que incluyeron sectores denudados, perfiles expuestos y un corral; no se realizaron hallazgos. Por último, aguas arriba del arroyo Los Loros a unos seis $\mathrm{km}$ del sitio y sobre un tributario del arroyo se relevó -sobre una morena (51'21' 16 , 54' 'S y $72^{\circ} 14^{\prime}$ 58, 09'" O)- una superficie de erosión $\left(8.000 \mathrm{~m}^{2}\right)$ donde tampoco se registraron materiales.

El estudio de los artefactos se realizó considerando las siguientes variables: tipo artefactual, materia prima empleada y calidad para la talla (sensu Aragón \& Franco, 1997), estado, dimensiones, presencia de talón y, para el caso de los artefactos formatizados, su tipo, la forma base y características generales. Se siguieron los lineamientos propuestos por Aschero (1975, revisión 1983). Se contabilizan exclusivamente aquellos desechos de talla que presenten talón.

Para cada uno de los puntos de recolección se discrimina la superficie en la que se encontraba disperso el material, su frecuencia separada en núcleos, desechos de talla y artefactos formatizados (Tablas 1 a 6).

\section{DISCUSIÓN y CONCLUSIONES}

Los materiales aquí presentados comparten las características de su ubicación (sector plano con cota igual o superior a los $300 \mathrm{~m}$, a un lado del valle en proximidades del bosque actual) con los sitios localizados sobre el

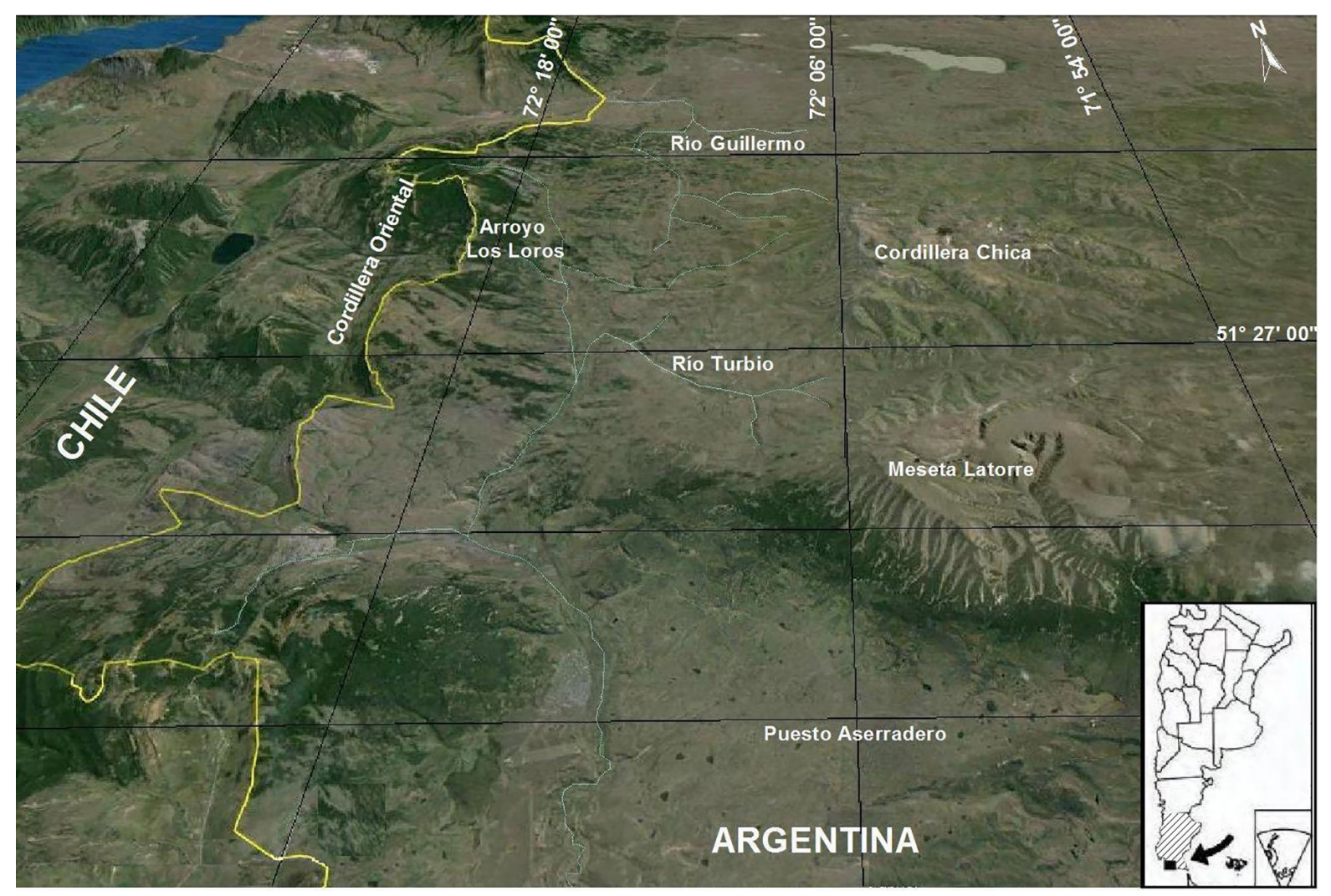

Fig. 1. Ubicación del arroyo Los Loros y demás espacios mencionados en el texto. 


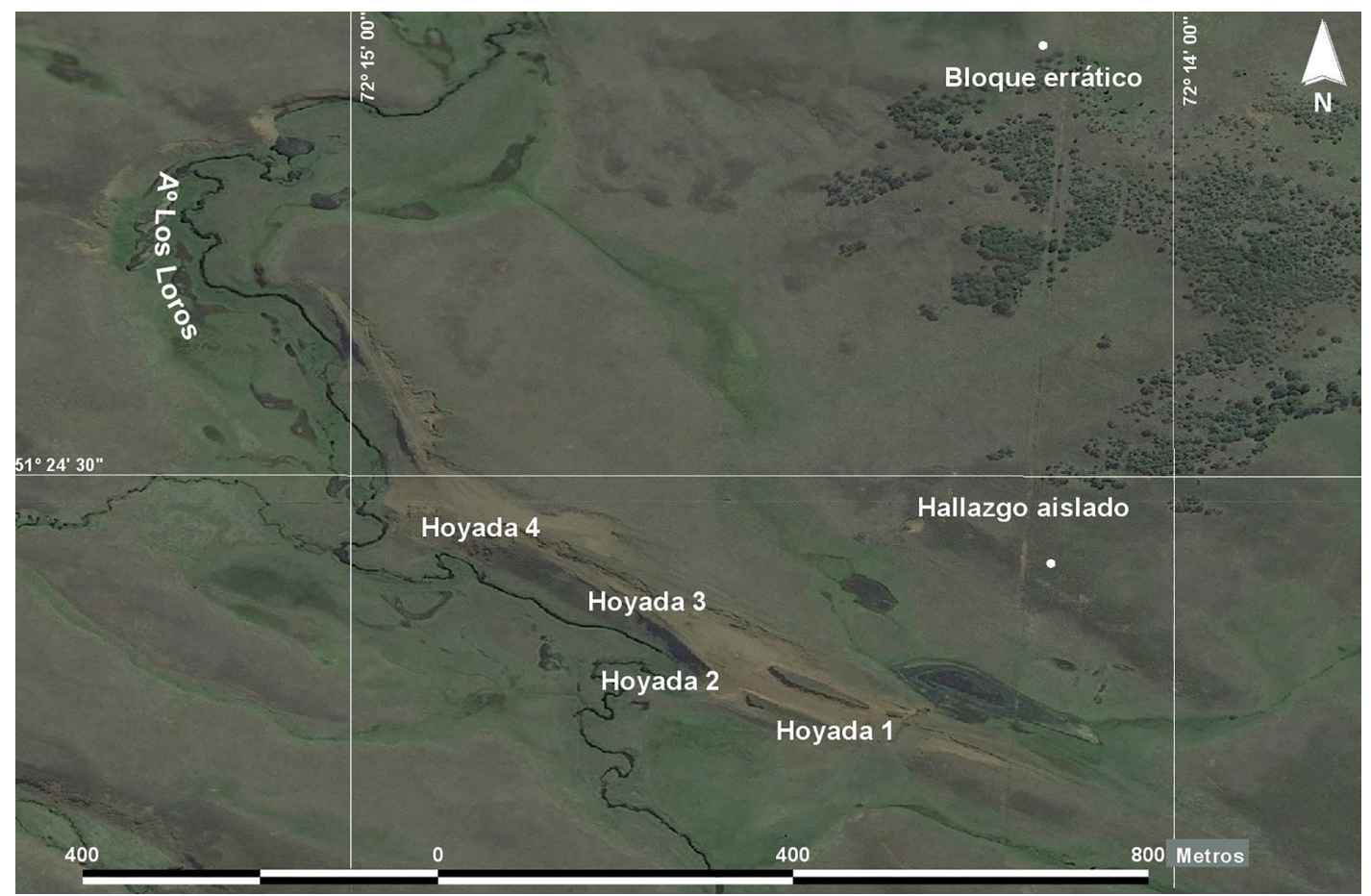

Fig. 2. Hoyadas del sitio Arroyo Los Loros, Bloque Errático y material aislado.
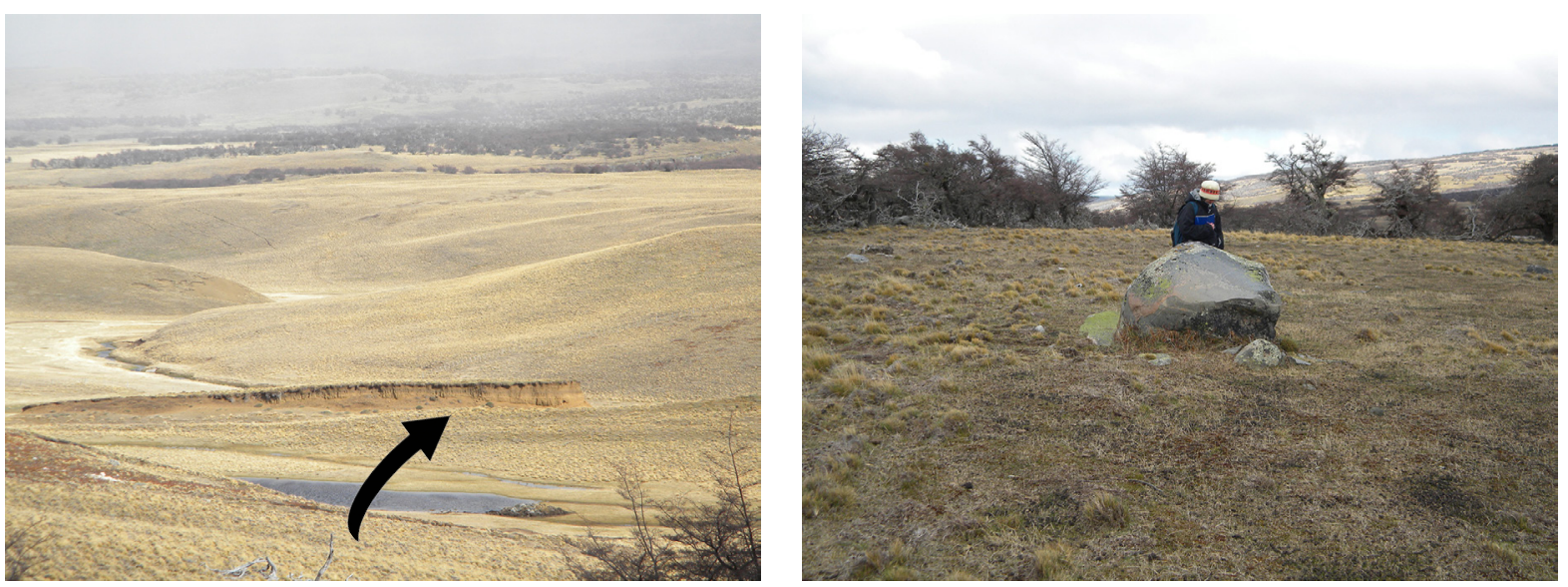

Figs. 3a. Vista norte del sitio Arroyo Los Loros. 3b. Bloque errático.

valle del río Guillermo (Pallo \& Borrero, 2015).

El registro de artefactos líticos del sitio Arroyo Los Loros muestra el aprovechamiento expeditivo de rocas inmediatamente disponibles, entre las que predominaron las lutitas negras de buena y muy buena calidad para la talla $(71,42 \%)$; lo que también se ve en los desechos de talla sin talón. La presencia de núcleos $(11,11 \%)$ -globulosos con extracciones multidireccionales y con reserva de corteza-, desechos de talla con corteza (28,57\%), el predominio de talones lisos y corticales (89\%), los bulbos marcados y los tamaños medianos y grandes de los artefactos sustentan la propuesta. Se destaca la presencia de raederas $(\mathrm{N}=4)$ que conservan potencial de uso. Ningún artefacto se relacionaría con el aprovechamiento del bosque en particular. Esta evidencia, junto con la baja frecuencia general de artefactos sugiere una muy baja intensidad de uso y de manera ocasional del 
Tabla 1. Sito Arroyo Los Loros. Hoyada 1. Frecuencia de tipos artefactuales. Referencias: L.: lasca, RSbV: rodado silíceo de base volcánica, MB: muy buena, B: buena. Los números entre paréntesis indican que la pieza se encuentra fracturada en esa dimensión. Además se registraron 11 lascas angulares de lutita B y seis lascas angulares de lutita MB (dos de módulo laminar), sin talón.

\section{HOYADA 1}

\begin{tabular}{|c|c|c|c|c|c|c|}
\hline \multicolumn{7}{|c|}{ Concentración $1\left(1 \mathrm{~m}^{2}\right)$} \\
\hline Tipo de artefacto & $\begin{array}{l}\text { Materia prima/ } \\
\text { Calidad }\end{array}$ & Largo $\mathrm{mm}$ & $\begin{array}{l}\text { Ancho } \\
\mathrm{mm}\end{array}$ & $\begin{array}{c}\text { Espesor } \\
\mathrm{mm}\end{array}$ & Talón & $\begin{array}{l}\text { Forma base/ } \\
\text { Observaciones }\end{array}$ \\
\hline L. angular & Lutita / B & 36 & 35 & 7,5 & Liso & Fractura en charnela \\
\hline L. angular & Lutita / B & 66,5 & 64 & 17,5 & Liso & --- \\
\hline L. angular & Lutita / B & 53,5 & 45,5 & 18 & Liso & --- \\
\hline \multicolumn{7}{|c|}{ Concentración $2\left(20 \mathrm{~m}^{2}\right)$} \\
\hline Núcleo & Lutita / MB & 95 & 70 & 33 & -- & $\begin{array}{l}5 \text { extracciones } \\
\text { multidireccionales, } \\
50 \% \text { de corteza }\end{array}$ \\
\hline Núcleo & $\mathrm{RSbV} / \mathrm{B}$ & 66 & 51 & 49 & --- & $\begin{array}{c}\text { Globuloso, } 7 \text { extracciones } \\
\text { multidireccionales, } 25 \% \text { de } \\
\text { corteza }\end{array}$ \\
\hline Chunk & Lutita / B & 54 & 34 & 20 & --- & -- \\
\hline L. primaria & $\mathrm{RSbV} / \mathrm{B}$ & 93 & 125 & 43 & Liso & Bulbo muy marcado \\
\hline L. primaria & $\mathrm{RSbV} / \mathrm{B}$ & 85 & 84 & 27,5 & Liso & --- \\
\hline L. primaria & $\mathrm{RSbV} / \mathrm{B}$ & 48,5 & 45 & 13 & Liso & Bulbo muy marcado \\
\hline L. primaria & Lutita / MB & 66,5 & 63 & 20 & Liso & Bulbo muy marcado \\
\hline L. primaria & Lutita / MB & 51 & 60 & 12 & Liso & --- \\
\hline L. secundaria & $\mathrm{RSbV} / \mathrm{B}$ & 50,5 & 75 & 36 & Liso & Bulbo muy marcado \\
\hline L. dorso & $\mathrm{RSbV} / \mathrm{B}$ & (58) & (72) & 12 & Cortical & --- \\
\hline L. dorso & Luita / MB & $(31,5)$ & $(54,5)$ & 11,5 & Cortical & Lasca adventicia \\
\hline L. dorso & Lutita / B & 42 & 69 & 11 & Cortical & --- \\
\hline L. angular & Lutita / B & 63 & (59) & 11 & Liso & --- \\
\hline L. angular & Lutita / MB & (38) & 57,5 & 15 & Liso & -- \\
\hline L. angular & Lutita / MB & 55 & 30 & 8 & Liso & Bulbo muy marcado \\
\hline L. angular & Lutita / MB & (25) & (50) & 8 & Liso & --- \\
\hline L. angular & Lutita / MB & (25) & (41) & 5 & Liso & --- \\
\hline L. angular & Lutita / MB & 31 & 32 & 10 & Liso & --- \\
\hline L. plana & Lutita / MB & (40) & (71) & 20 & Liso & --- \\
\hline L. plana & $\mathrm{RSbV} / \mathrm{B}$ & (41) & $(59,5)$ & 9 & Liso & Lasca adventicia \\
\hline L. plana & Lutita / MB & 45 & $(51,5)$ & 11 & Liso & --- \\
\hline L. plana & Lutita / MB & (25) & (41) & 8,5 & Liso & --- \\
\hline L. plana & Lutita / MB & (21) & 44,5 & 7,5 & Diedro & -- \\
\hline L. plana & Lutita / MB & (21) & (34) & 6 & Liso & --- \\
\hline L. plana & Lutita / MB & (25) & (30) & 3 & Liso & --- \\
\hline L. plana & Lutita / MB & $(22,5)$ & $(23,5)$ & 2,5 & Liso & Lasca adventicia \\
\hline \multicolumn{7}{|c|}{ Interconcentración } \\
\hline L. angular & Lutita / MB & 15 & 16,5 & 7 & Liso & --- \\
\hline
\end{tabular}


Tabla 2. Sito Arroyo Los Loros. Hoyada 2. Frecuencia de tipos artefactuales.

Referencias: L.: lasca, MB: muy buena, B: buena. Los números entre paréntesis indican que la pieza se encuentra fracturada en esa dimensión.

\begin{tabular}{|c|c|c|c|c|c|c|}
\hline \multicolumn{7}{|c|}{ HOYADA 2} \\
\hline \multicolumn{7}{|c|}{ Concentración $1\left(49 \mathrm{~m}^{2}\right)$} \\
\hline Clase artefactual & $\begin{array}{l}\text { Materia prima/ } \\
\text { Calidad }\end{array}$ & Largo $\mathrm{mm}$ & $\begin{array}{l}\text { Ancho } \\
\mathrm{mm}\end{array}$ & $\begin{array}{l}\text { Espesor } \\
\mathrm{mm}\end{array}$ & Talón & $\begin{array}{l}\text { Forma base/ } \\
\text { Observaciones }\end{array}$ \\
\hline Núcleo & Lutita / MB & 110 & 72 & 72 & --- & $\begin{array}{c}\text { Globuloso, } 9 \text { extracciones } \\
\text { multidireccionales, entre un } 25 \% \text { y } \\
\text { un } 50 \% \text { de corteza }\end{array}$ \\
\hline Núcleo & Lutita / MB & 69 & 49 & 26 & --- & $\begin{array}{l}3 \text { extracciones, } \\
50 \% \text { de corteza }\end{array}$ \\
\hline L. secundaria & Lutita / MB & 50,5 & 44 & 15 & Liso & Bulbo muy marcado \\
\hline L. secundaria & Lutita / MB & 56 & 57 & 19 & Liso & --- \\
\hline L. dorso & Lutita / MB & 60 & 52 & 6 & Liso & --- \\
\hline L. angular & Lutita / MB & 75 & 46 & 11 & Liso & Bulbo muy marcado \\
\hline L. angular & Lutita / MB & 53 & 51 & 14 & Liso & Bulbo muy marcado \\
\hline L. angular & Lutita / MB & 65 & 38 & 6,5 & Liso & Lasca adventicia \\
\hline L. angular & Lutita / MB & 54 & 47 & 17 & Liso & $25 \%$ de corteza \\
\hline L. angular & Basalto / B & 43 & 37 & 9 & Diedro & $25 \%$ de corteza \\
\hline \multicolumn{7}{|c|}{ Concentración $2\left(20 \mathrm{~m}^{2}\right)$} \\
\hline L. primaria & Lutita / MB & 82 & 54 & 22 & Cortical & $\begin{array}{l}\text { Bulbo muy marcado y } \\
\text { fractura en charnela }\end{array}$ \\
\hline L. secundaria & Lutita / MB & 64 & 62 & 20 & Liso & $\begin{array}{l}\text { Bulbo muy marcado y } \\
\text { lasca adventicia }\end{array}$ \\
\hline L. angular & Lutita / MB & 54 & 55,5 & 9 & Liso & Lasca adventicia \\
\hline
\end{tabular}

sitio por parte de las poblaciones humanas. Las mismas apreciaciones caben para los materiales observados en el Bloque errático. La escasez de la categoría hallazgos aislados habla de lo efímero de las ocupaciones en la región. A la vez, dada la ubicación occidental del arroyo Los Loros, -dentro de una zona caracterizada como de estrés invernal (Sturzenbaum \& Oliva, 2001; ver Pallo, 2012)- el uso de este espacio habría sido estacional (primavera-verano).

Toda esta evidencia abona la discusión en torno al papel de corredor postulado para el valle del río Guillermo (Pallo \& Borrero, 2015). En este caso, el valle del río habría permitido la circulación de poblaciones en sentido OesteEste. Los materiales recuperados en el sitio del valle medio del arroyo Los Loros complementan esta problemática al brindar evidencia empírica que permite hipotetizar también la existencia de un corredor de fácil tránsito complementario al del río Guillermo, pero que habría funcionado en sentido Norte-Sur (desde el valle del río Guillermo, conectando con el valle del arroyo Los Loros y a este con el valle del río Turbio) (Fig. 1). Al igual que el planteo realizado para el caso del río Guillermo, la mayoritaria utilización de la lutita, la presencia de módulos laminares -si bien en muy bajo número- (ver Tabla 4) y las bajas frecuencias artefactuales, sustentan la conexión de este espacio con sectores occidentales. Así, el corredor Los Loros-Turbio, enmarcado por el contrafuerte Oeste de la Cordillera Chica y la Meseta Latorre y el lado Este de la Cordillera Oriental (límite internacional) apoyarían también la conexión propuesta entre el valle del río Guillermo y Puesto Aserradero (Charlin et al. 2011; Pallo \& Borrero, 2015), ubicado hacia el Sur de la Meseta Latorre (Fig. 1). El planteo se ve también respaldado por los resultados distribucionales de 
Tabla 3. Sito Arroyo Los Loros. Hoyada 3. Frecuencia de tipos artefactuales. Referencias: L.: lasca, FAF: fragmento de artefacto formatizado, RSbV: rodado silíceo de base volcánica, MB: muy buena, B:

buena. Los números entre paréntesis indican que la pieza se encuentra fracturada en esa dimensión. Además, se registró una lasca indiferenciada de lutita $\mathrm{MB}$, una lasca indiferenciada de madera silicificada $\mathrm{MB}$, tres lascas angulares de lutita MB y una lasca secundaria de RSbV B sin talón.

\begin{tabular}{|c|c|c|c|c|c|c|}
\hline \multicolumn{7}{|c|}{ HOYADA 3} \\
\hline \multicolumn{7}{|c|}{ Concentración $1\left(4 \mathrm{~m}^{2}\right)$} \\
\hline Clase artefactual & $\begin{array}{l}\text { Materia prima/ } \\
\text { Calidad }\end{array}$ & Largo mm & $\begin{array}{c}\text { Ancho } \\
\mathrm{mm}\end{array}$ & $\begin{array}{l}\text { Espesor } \\
\mathrm{mm}\end{array}$ & Talón & $\begin{array}{l}\text { Forma base/ } \\
\text { Observaciones }\end{array}$ \\
\hline L. secundaria & Lutita / MB & 75 & 75 & 18,5 & Diedro & --- \\
\hline L. angular & Lutita / MB & 79 & 88 & 27 & Liso & Retoque de $35 \mathrm{~mm}$ \\
\hline L. angular & M. silicificada / MB & 91,5 & 64 & 11 & Liso & -- \\
\hline \multicolumn{7}{|c|}{ Concentración $2(20$ m²) } \\
\hline Núcleo & $\mathrm{RSbV} / \mathrm{B}$ & 81 & 67 & 41 & -- & $\begin{array}{c}\text { Globuloso, } 6 \text { extracciones multidireccionales, } \\
25 \% \text { de corteza }\end{array}$ \\
\hline Núcleo & Lutita / MB & 95 & 39 & 29 & -- & 3 extracciones paralelas cortas (34 mm) \\
\hline L. primaria & $\mathrm{RSbV} / \mathrm{B}$ & 36 & 43 & 12,5 & Liso & -- \\
\hline L. secundaria & Lutita / MB & 36,5 & 16 & 12 & Puntiforme & -- \\
\hline L. dorso & $\mathrm{RSbV} / \mathrm{B}$ & 31,5 & 37 & 5 & Liso & -- \\
\hline L. angular & Lutita / B & $(29,5)$ & (24) & 7 & Liso & -- \\
\hline L. angular & Lutita / MB & 43,5 & 39 & 6 & Liso & Lasca adventicia \\
\hline L. angular & Lutita / MB & 30 & 51 & 10 & Estallado & -- \\
\hline L. angular & Lutita / MB & 22 & 34,5 & 9,5 & Liso & -- \\
\hline \multicolumn{7}{|c|}{ Interconcentraciones } \\
\hline Núcleo & Dacita / MB & 55 & 42 & 32,5 & - & $\begin{array}{c}\text { Globuloso, } 8 \text { extracciones multidireccionales y } \\
25 \% \text { de corteza }\end{array}$ \\
\hline L. angular & M. silicificada / MB & (36) & 37 & 14 & Liso & -- \\
\hline Raedera & Dacita / MB & (62) & 66 & 21,5 & Liso & $\begin{array}{l}\text { Sobre lasca angular, } 1 \text { hilera de lascado, } \\
\text { retoque, bulbo muy marcado, lasca adventicia } \\
\text { y potencial de reactivación }\end{array}$ \\
\hline Raedera & Lutita / MB & 54 & 80 & 18 & Liso & $\begin{array}{l}\text { Sobre lasca angular, } 2 \text { hileras de lascados, } \\
\text { retoque, módulo corto ancho y potencial de } \\
\text { reactivación }\end{array}$ \\
\hline FAF & Basalto / MB & $(40)$ & (54) & 12 & - & $\begin{array}{c}\text { Sobre lasca angular, } 1 \text { hilera de lascado, } \\
\text { retoque }\end{array}$ \\
\hline
\end{tabular}

un sector del ecotono bosque-estepa llevados a cabo hacia el Noreste, también en campos de la Ea. Cancha Carrera, los que mostraron, a pesar de la buena visibilidad arqueológica y lo intenso de los muestreos realizados, la brusca disminución de las frecuencias artefactuales al alejarse de los cursos de agua (Carballo Marina \& Madrid, 2011).

La información presentada no sólo suma nueva evidencia a lo conocido para la cuenca superior del río Gallegos (Borrero \& Borrazzo,
2011) sino que también colabora con la discusión del uso humano del bosque en el pasado. Si bien la evidencia del valle medio del arroyo Los Loros se diferencia de las registradas en regiones ubicadas hacia el Norte como el lago Argentino (Borrero \& Muñoz, 1999), el lago Viedma y el lago San Martín (Belardi \& Carballo Marina, 2014) -donde por la presencia del Campo de Hielo Patagónico Sur no se puede circular hacia el Oeste-, es concordante en cuanto a la baja intensidad de uso de dicho ambiente del Sur de Patagonia. 
Tabla 4. Sito Arroyo Los Loros. Hoyada 4. Frecuencia de tipos artefactuales.

Referencias: L.: lasca, RSbV: rodado silíceo de base volcánica, MB: muy buena, B: buena.

\begin{tabular}{|c|c|c|c|c|c|c|}
\hline \multicolumn{7}{|c|}{ HOYADA 4} \\
\hline \multicolumn{7}{|c|}{ Hallazgos aislados } \\
\hline Clase artefactual & $\begin{array}{c}\text { Materia prima/ } \\
\text { Calidad }\end{array}$ & $\begin{array}{l}\text { Largo } \\
\mathrm{mm}\end{array}$ & $\begin{array}{l}\text { Ancho } \\
\mathrm{mm}\end{array}$ & $\begin{array}{c}\text { Espesor } \\
\mathrm{mm}\end{array}$ & Talón & $\begin{array}{l}\text { Forma base/ } \\
\text { bservaciones }\end{array}$ \\
\hline L. angular & $\mathrm{RSbV} / \mathrm{R}$ & 65 & 43 & 23 & Cortical & $25 \%$ de corteza \\
\hline L. angular & Lutita / MB & 94 & 61 & 14 & Diedro & Extracciones laminares paralelas \\
\hline Raedera & Andesita / B & 121 & 91 & 25,5 & Cortical & $\begin{array}{c}\text { Sobre lasca angular, } 2 \text { hileras de } \\
\text { lascados, retoque, } 25 \% \text { de corteza, } \\
\text { módulo corto ancho, potencial de } \\
\text { reactivación }\end{array}$ \\
\hline
\end{tabular}

Tabla 5. Hallazgo aislado. Referencia: B: buena.

\begin{tabular}{|c|c|c|c|c|c|c|}
\hline \multicolumn{7}{|c|}{ Hallazgo aislado } \\
\hline Clase artefactual & $\begin{array}{c}\text { Materia prima/ } \\
\text { Calidad }\end{array}$ & $\begin{array}{c}\text { Largo } \\
\mathrm{mm}\end{array}$ & $\begin{array}{l}\text { Ancho } \\
\mathrm{mm}\end{array}$ & $\begin{array}{c}\text { Espesor } \\
\mathrm{mm}\end{array}$ & Talón & $\begin{array}{l}\text { Forma base/ } \\
\text { Observaciones }\end{array}$ \\
\hline Núcleo & Basalto / B & 135 & 95 & 61 & --- & $\begin{array}{c}\text { Globuloso, } \\
8 \text { extracciones multidireccionales, } \\
25 \% \text { de corteza }\end{array}$ \\
\hline
\end{tabular}

Tabla 6. Bloque errático. Frecuencia de tipos artefactuales. Referencias: L.: lasca, RSbV: rodado silíceo de base volcánica, MB: muy buena, B: buena. Además, se registró una lasca angular basalto B y una lasca angular en lutita B sin talón.

\begin{tabular}{ccccccc}
\hline & \multicolumn{5}{c}{ Bloque errático $\left(1 \mathrm{~m}^{2}\right)$} \\
Clase artefactual & $\begin{array}{c}\text { Materia prima/ } \\
\text { Calidad }\end{array}$ & $\begin{array}{c}\text { Largo } \\
\mathrm{mm}\end{array}$ & $\begin{array}{c}\text { Ancho } \\
\mathrm{mm}\end{array}$ & $\begin{array}{c}\text { Espesor } \\
\mathrm{mm}\end{array}$ & Talón & $\begin{array}{c}\text { Forma base/ } \\
\text { Observaciones }\end{array}$ \\
\hline Núcleo & RSbV / B & 87 & 76 & 46 & -- & $\begin{array}{c}\text { Globuloso, } 7 \text { extracciones } \\
\text { multidireccionales, } 50 \% \text { de corteza }\end{array}$ \\
L. primaria & Lutita / MB & 25 & 34 & 5,5 & Cortical & Lasca adventicia \\
L. secundaria & Basalto / MB & 63,5 & 45 & 17 & Cortical & Bulbo muy marcado \\
L. secundaria & Lutita / MB & 21 & 22 & 5 & Cortical & --- \\
\hline L. dorso & Basalto / MB & 76 & 59 & 20 & Cortical & Bulbo muy marcado
\end{tabular}

\section{AGRADECIMIENTOS}

Al Lic. Matías Ambasch por su gentileza al brindarnos información de la zona y al Lic. Pedro Tiberi por la confección de los mapas. A los dos evaluadores por sus comentarios.

Los trabajos fueron realizados en el marco del PIP CONICET 0262, "Caracterización y comparación de los modos de interacción y circulación humana al norte y sur del estrecho de Magallanes".

\section{BIBLIOGRAFÍA}

Aschero, C. (1975), (revisión 1983). Ensayo para una clasificación morfológica de artefactos líticos. MS.

Aragón, E., \& Franco N. V. (1997). Características de rocas para la talla por percusión y propiedades petrográficas. Anales del Instituto de la Patagonia, Serie Ciencias Humanas, 25, 187-200.

Belardi, J. B., \& Carballo Marina, F. (2014). La señal arqueológica del interior del bosque en la margen sur del lago San Martín (provincia de Santa Cruz). 
Comechingonia, 18(2), 181-202.

Borrero, L. A., \& Muñoz, A. S. (1999). Tafonomía en el bosque patagónico. Implicaciones para el estudio de su explotación y uso por poblaciones humanas de cazadores-recolectores. En Soplando en el viento. Actas de las III Jornadas de Arqueología de la Patagonia (pp. 43-56). San Carlos de Bariloche, Instituto Nacional de Antropología y Pensamiento Latinoamericano y Universidad Nacional del Comahue.

Borrero, L. A., \& Borrazzo, K. (2011). La geografía cultural del sudoeste de Patagonia continental. En L. A. Borrero \& K. Borrazzo (Comp.), Bosques, montañas y cazadores. Investigaciones Arqueológicas en Patagonia Meridional (pp. 7-36). Buenos Aires, CONICET-IMHICIHU, Impresiones Dunken.

Carballo Marina, F., \& Madrid, P. (2011). Estudio de impacto ambiental sobre el tendido interconexión Tramo La Esperanza- Río Gallegos y Tramo La Esperanza- Río Turbio (Departamento de Güer Aike, provincia de Santa Cruz): el registro arqueológico. Informe presentado a Transportel Patagónica SA.

Charlin, J,, Borrero, L., \& Pallo, C. (2011). Ocupaciones humanas en el área Noroocidental del río Gallegos (Prov. de Santa Cruz, Argentina). En L. A. Borrero y K. Borrazzo (Compiladores), Bosques, Montañas y Cazadores. Investigaciones arqueológicas en Patagonia Meridional (pp. 179-210). Buenos Aires, CONICET-IMHICIHU, Impresiones Dunken.

García, J. L., Hall, B., Kaplan, M. R., Vega, R. M., \& Strelin, J. A. (2014). Glacial geomorphology of the Torres del Paine region (southern Patagonia): Implications for glaciation, deglaciation and paleolake history. Geomorphology, 204, 599-616.

Ibar Sierra, E. (2002) [1877]. Relación de los hechos en el Estrecho de Magallanes I la Patagonia Austral durante los últimos meses de 1877, por el Ayudante del Museo Nacional de Chile, don Enrique Ibar Sierra. En Martinic, M. (Ed.), Marinos de a caballo. Exploraciones terrestres de la Armada de Chile en la Patagonia austral y la Tierra del Fuego 1877. 1897 (pp. 19-60). Punta Arenas, Universidad de Magallanes y Universidad de Playa Ancha.

Legoupil, D. (2009). La ocupación del alero Cerro Castillo en la antigua cuenca glaciar del Lago Toro hacia el V milenio AP. Magallania, 37(1), 19-38.

Pallo, M. C. (2012). El estrés invernal como generador de áreas marginales en el extremo sur de Patagonia continental durante el Holoceno tardío. Comechingonia Virtual VI (1), 86-114.

Pallo, C., \& Borrero, L. A. (2015). Arqueología de corredores boscosos en Patagonia meridional: el caso del río Guillermo (SO de la provincia de Santa Cruz, Argentina). Intersecciones en Antropología, 16(2), 313-326.

Peri, P., Martínez Pastur, G., Monelos, L., \& Beroiz, M. (2013). La distribución continental más oriental de Nothofagus antárctica en el río Gallegos (Santa Cruz). Anales del Instituto de la Patagonia, 44(1), 113-117.

Sturzenbaum, P., \& Oliva, G. (2001). Manejo de riesgos climáticos. En P. Borreli y G. Oliva (Eds.), Ganadería Ovina Sustentable en la Patagonia Austral. Tecnologías de Manejo Extensivo (pp. 255-270). Santa Cruz y Buenos Aires, INTA y ErreGé. 
